\title{
Coincidental detection of the first outbreak of carbapenemase-producing Klebsiella pneumoniae colonisation in a primary care hospital, Finland, 2013
}

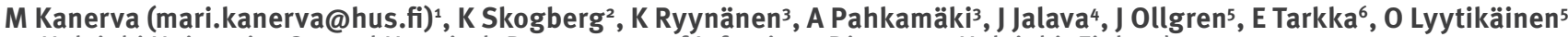

1. Helsinki University Central Hospital, Department of Infectious Diseases, Helsinki, Finland

2. Helsinki University Central Hospital, Department of Infectious Diseases, Espoo, Finland

3. Espoo Hospital, Espoo, Finland

4. National Institute for Health and Welfare, Department of Infectious Diseases Surveillance and Infection Control, Antimicrobial Resistance Unit, Turku, Finland

5. National Institute for Health and Welfare, Department of Infectious Diseases Surveillance and Infection Control, Epidemiologic Surveillance and Response Unit, Helsinki, Finland

6. HUSLAB, Department of Bacteriology, Helsinki, Finland

Kanerva M, Skogberg K, Ryynänen K, Pahkamäki A, Jalava J, Ollgren J, Tarkka E, Lyytikäinen O. Coincidental detection of the first outbreak of carbapenemaseproducing Klebsiella pneumoniae colonisation in a primary care hospital, Finland, 2013. Euro Surveill. 2015;20(26):pii=21172. Available online: http://www. eurosurveillance.org/ViewArticle.aspx?Articleld=21172

In Finland, occurrence of Klebsiella pneumoniae carbapenemase-producing $K$. pneumoniae (KPC-KP) has previously been sporadic and related to travel. We describe the first outbreak of colonisation with KPC-KP strain ST512; it affected nine patients in a 137-bed primary care hospital. The index case was detected by chance when a non-prescribed urine culture was taken from an asymptomatic patient with suprapubic urinary catheter in June 2013. Thereafter, all patients on the 38 -bed ward were screened until two screening rounds were negative and extensive control measures were performed. Eight additional KPC-KP-carriers were found, and the highest prevalence of carriers on the ward was nine of 38 . All other patients hospitalised on the outbreak ward between 1 May and 10 June and 101 former roommates of KPC-KP carriers since January had negative screening results. Two screening rounds on the hospital's other wards were negative. No link to travel abroad was detected. Compared with non-carriers, but without statistical significance, KPC-KP carriers were older ( 83 vs 76 years) and had more often received antimicrobial treatment within the three months before screening ( $9 / 9$ vs $90 / 133$ ). No clinical infections occurred during the six-month follow-up. Early detection, prompt control measures and repetitive screening were crucial in controlling the outbreak.

\section{Introduction}

Carbapenemase-producing Enterobacteriaceae (CPE) pose a severe health threat causing potentially incurable infections because antimicrobial treatment options are limited due to the resistance. In the United States (US), Klebsiella pneumoniae carbapenemase (KPC) is the most common mechanism of carbapenemase resistance among Enterobacteriaceae, while metallo-beta-lactamases such as VIM, IMP and NDM are common especially in Asia [1]. Plasmid-encoded KPC was first identified in North Carolina in 1996 and has been increasingly reported worldwide, including in Israel since 2005 [1-3]. In the US, KPC strains first spread in short-stay acute care hospitals. Subsequently, KPCproducing Enterobacteriaceae were recognised in longterm care facilities and in a study in $2010 / 11$, up to $30 \%$ colonisation prevalence has been detected e.g. in longterm acute care hospitals [4].

In 2013, most European countries detected CPE: nine countries reported sporadic cases or single or sporadic hospital outbreaks, 11 reported regional or national spread and in Greece, Italy and Malta, CPE were regularly isolated in most hospitals and have become endemic [5]. Most outbreak reports before 2013 were from acute care hospitals [6-8]. The source of CPE in the index case is described only in some of the reports and was typically related to hospital transfers from abroad $[6,8,9]$. Also in the Nordic countries, sporadic cases of CPE have been documented among repatriated hospital patients, causing some local spread or sporadic hospital outbreaks [10-12]. In Finland, transmission of CPE between two patients was suspected only once, in 2011 [11].

Here, we present the first outbreak in a Finnish primary care hospital in summer 2013 with nine patients colonised with KPC-producing K. pneumoniae (KPC-KP) strain $\mathrm{ST}_{512}$. We describe the outbreak control measures and risk factors for KPC-KP carriage. 


\section{Methods}

\section{Setting}

The outbreak occurred in a 137 -bed primary care hospital (Hospital A) in the Helsinki metropolitan area, Finland, responsible for post-acute care of a catchment population of 250,000 . In addition, the area is served by a second hospital with tertiary care wards and three primary care wards (Hospital B). These wards provide acute care and transfer patients to Hospital $A$ which has four wards: Ward A with 38 beds for geriatric patients, Ward B with 38 beds for orthogeriatric patients and rehabilitation, Ward C with 27 beds for rehabilitation of patients with cerebral insults and Ward D with 34 beds for terminal care and geriatric patients. Patients with carriage of multidrug-resistant organisms (MDRO; mainly meticillin-resistant Staphylococcus aureus (MRSA)) in this hospital are treated at Ward $A$ in contact isolation. Usually, none or only few such patients are present and the ward therefore accepts also non-carriers in dedicated rooms to avoid empty beds.

\section{Index case}

The index case was detected on Ward A on 10 June 2013 after a urine specimen was taken for culture from an asymptomatic patient who had been staying on that ward since February 2013. A nurse took the sample without a prescription because the patient's catheter urine looked turbid. The culture grew K. pneumoniae resistant to meropenem. The suprapubic catheter had been inserted nine days earlier during an outpatient visit to the tertiary care hospital due to urinary obstruction. An earlier urine culture from this patient in March had been negative (information on the clinical indication for that culture is not available).

\section{Screening and contact tracing}

After the detection of the index case, all patients on Ward A were screened for KPC-KP. Screening was repeated weekly until there were no new positive KPC-KP findings on the ward for two consecutive screening rounds, thereafter monthly until all known KPC-KP-carriers were discharged. In addition, the three other wards of Hospital A were screened twice, one ward in Hospital B was screened once and three primary care wards at Hospital $B$ that had frequent patient transfers to Ward A (Hospital A) were screened twice.

All patients who had been on Ward A since 1 May 2013 or been roommates of KPC-KP carriers in January 2013 or later (until 10 June 2013) were defined as exposed and thus screened. Discharged patients were screened as outpatients. Those who had been in other rooms than the KPC-KP carriers but on the same ward between January and April were flagged to be screened at a new admission. Altogether, 101 exposed patients were identified. Two negative sets of screening specimens at least one week after the end of exposure were required to rule out KPC-KP.
All KPC-KP carriers and their family members were interviewed about previous stays in hospitals abroad. The stays and room locations of all KPC-KP carriers at different hospitals and wards during the preceding six months were carefully evaluated to identify potential transmission links. The laboratory results of patients hospitalised concurrently in the same hospital were reviewed for findings of multidrug-resistant Enterobacteriaceae.

A screening set included stool or rectal swab, swabs of chronic wounds and catheter urine. Screening for the presence of multidrug-resistant Enterobacteriaceae was done by plating specimens on selective chromogenic KPC plates (CHROMagar, France). Isolates were identified by matrix-assisted laser desorption/ionisation time-of-flight (MALDI-TOF) mass spectrometry (VITEK MS, bioMeriéux), and antimicrobial susceptibilities were determined by disc diffusion method according to standards of the European Committee on Antimicrobial Susceptibility Testing (EUCAST) [13] or by gradient MIC determination (Etest, bioMeriéux). For isolates with reduced susceptibility to any carbapenem, the presence of carbapenemase genes was analysed by multiplex real-time PCR [14]. The strains possessing carbapenemase genes were subjected to multilocus sequence typing (MLST) and pulsed-field gel electrophoresis (PFGE) [11].

\section{Other infection control measures and environmental cultures}

The infection control teams of the primary and tertiary care hospitals worked closely together. KPC-KP carriers and patients exposed to KPC-KP were transferred into single rooms or cohorts, treated in contact isolation and flagged in the electronic patient charts at both hospitals. Ward A was closed for new admissions from 19 June to 26 July 2013.

Infection control measures (hand hygiene, standard precautions and contact isolation) at Ward A were promoted by a lecture given by an infectious diseases specialist and frequent visits by the infection control nurse to the ward meetings up to two or three times a week during the summer. In addition, there were three summarising meetings with the infection control team and the hospital administration. Healthcare workers' hands were inspected for rings, bracelets and chronic wounds, and compliance with hand hygiene and environmental cleaning procedures was monitored by the infection control nurse during the ward visits. The use of the disinfecting washing machine, washing room cleaning and disinfection (especially the bunk and shower chairs) were reviewed and written instructions were provided. Only permanent and trained cleaners were allowed to work at Ward A. Hypochlorite disinfectant was introduced for daily surface cleaning in the rooms of KPC-KP carriers and the washing room. The indications to use urinary catheters and antimicrobial practices were reviewed. 
Duration and location of hospital stays of patients colonised with Klebsiella pneumoniae carbapenemase-producing K. pneumonia, Finland, $2013(\mathrm{n}=9)$
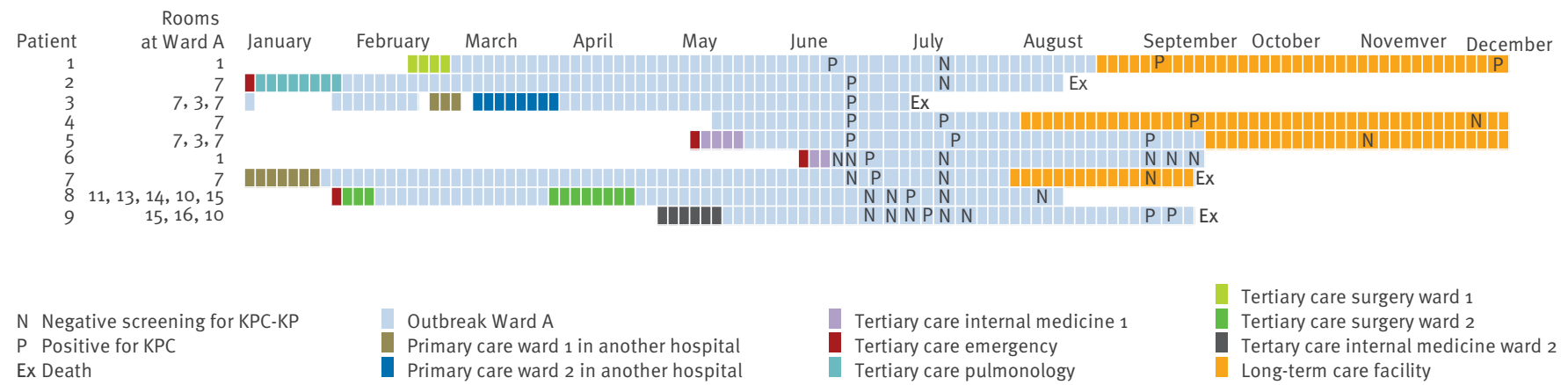

Tertiary care internal medicine 1

Tertiary care emergency

Tertiary care pulmonology
Tertiary care surgery ward

Tertiary care surgery ward 2

Tertary care internal medicine ward 2

Long-term care facility

KPC-KP: Klebsiella pneumoniae carbapenemase-producing $K$. pneumoniae.

The date of KPC-KP detection and positive follow-up screenings are marked with $\mathrm{P}$ and the dates of negative screening results are marked with $\mathrm{N}$. Colours indicate the various wards a patient was staying on.

Altogether, 10 culture swabs were taken at Ward A from various surfaces frequently touched and thus suspected to be contaminated, e.g. in patient rooms and in bathrooms.

\section{Risk factor analysis}

To identify risk factors for KPC-KP carriage, a casecohort study was performed. The charts of all patients (nine KPC-KP carriers and 133 non-carries) present at the four wards of Hospital A on the day of the first screening round during 14 to 17 June were reviewed by three doctors, MK, KS and KR. Analyses were performed with SPSS (version 20.0 Chicago, IL, US). Differences in the frequency of dichotomic variables between KPC-KP carriers and non-carriers were compared by Fisher's exact test and the means of continuous variables were compared by Mann-Whitney and Kruskal-Wallis tests. Multivariable logistic regression analysis was done with backward selection using Akaike information criteria (AIC).

\section{Results}

KPC-KP screening and contact tracing

After the detection of the index case, the first screening round at Ward A revealed four KPC-KP-carriers in a four-bed room that was used for five patients at the time. In each of the four following weekly rounds, one additional KPC-KP carrier was identified (Figure): Patient 6 was a roommate of the index case, Patient 7 was the fifth patient in the overcrowded four-bed room, and the last two patients (number 8 and 9) were in two additional rooms. Patients 6 to 9 had two, one, two and three negative screening results, respectively, before becoming positive. No new KPC-KP were detected in the sixth and seventh screening rounds. The highest prevalence of KPC-KP carriers on Ward A was nine of 38 . Specimens obtained during screening as well as clinical specimens obtained at other wards
(B, $C$ and $D$ in Hospital $A$ ) and at Hospital $B$ ) revealed no KPC-KP-carriers. Altogether, 535 screening specimens were collected between 14 June and 2 August in seven screening rounds on Ward $A$ and two screening rounds on Wards $B, C$ and $D$.

No additional KPC-KP-carriers were found among the 101 exposed patients: Those still present on Ward $A$ $(n=18)$ were included in the screening rounds there and those transferred to long-term care facilities $(n=27)$ or discharged home $(n=37)$ were screened as outpatients. Results for all of them were negative until the end of October 2013. Nineteen of the exposed patients had died before screening.

Apart from two patients with KPC-KP only in catheter urine, all nine positive patients had KPC-KP in stools or rectal swab and one also in a sacral wound. The follow-up screenings showed that four patients spontaneously lost KPC-KP-carriage within one month, while four had persistent or intermittent carriage for at least three months (Figure). One KPC-KP carrier died before any follow-up screening could be done. None of the KPC-KP carriers developed clinical infection during a six-month follow-up period. By the end of September 2013, four of the nine KPC-KP carriers had died due to unrelated underlying diseases, two of them while still on Ward $A$. Of the nine, four had been admitted to a long-term care facility and three were discharged home.

None of the nine KPC-KP-carriers had been travelling abroad during the year before the outbreak. None of their family members had been hospitalised abroad. All but two of the KPC-KP-carriers had contact to Hospital $B$, neither at an outpatient visit during their stay in primary care nor at a direct admission. Review of the charts from patients staying at the same time on the same wards in Hospital B as the KPC-KP-carriers did 
not provide indications as to the origin of the KPC-KP outbreak either.

\section{Microbiological characterisation of isolates} All nine KPC-KP isolates represented the same MLST type ST512 (a single locus variant of ST258). PFGE analyses showed that all the strains were closely related. Seven strains had identical PFGE profiles (PFGE type A). Two strains, isolated four and 10 days after the first strain, had minor differences compared with the PFGE type $A$, i.e. PFGE type $A 1$ differed in three bands (of 21 bands seen in the PFGE gel) from type $A$ and PFGE type A2 differed in two bands (of 21 bands seen in the PFGE gel) from type $A$. In addition, types $A_{1}$ and $A_{2}$ differed from each other in four bands.

The isolates were resistant to all beta-lactams (all had a minimum inhibitory concentration (MIC) for meropenem of $>32 \mathrm{mg} / \mathrm{L}$ ): mecillinam, nitrofurantoin, ciprofloxacin, tobramycin, amikacin and trimethoprim. Their susceptibility varied for gentamicin, fosfomycin, tigecycline, sulfadiazine/trimethoprim and colistin. At the presence of $1 \mu \mathrm{g} / \mathrm{mL}$ rifampicin, the colistin MICs were reduced by a factor of 4 or 8 , which rendered isolates in eight patients potentially sensitive to colistin $(M I C<2)$ (data not shown).

\section{Other infection control measures and environmental cultures}

No skin breaks, rings or bracelets were found on the hands of healthcare workers. The ward audits revealed weaknesses in the placement of hand rub dispensers, the placement of clean urinary bottles in the toilets and in the cleaning of bathrooms. During the years 2012 and 2013, the use of alcohol hand rub had increased from 26.5 to $40.2 \mathrm{~L}$ per 1,000 patient-days at ward A. None of the 10 surface swabs was culture-positive for KPC-KP.

\section{Risk factors for KPC-KP}

All nine KPC-KP carriers were older than 70 years and had underling illnesses (eight patients with McCabe score $\geq 2$ [15]) (Table). Three were receiving antimicrobial treatment on the day the sample for their positive test was taken, one of which was considered inappropriate by the authors when reviewed for this study. KPC-KP carriers were more likely to have received antimicrobial treatment within three months than non-carriers (9/9 vs 90/133). The last date of antibiotic treatment was available for those who had stopped the treatment before screening, namely six of the nine carriers and 63 of the 133 non-carriers. It was more recent among the carriers than among non-carriers, a mean of 40 and 77 days before the first screening, respectively. Several of the carriers had multiple risk factors for contaminating their surroundings (Table): of those, six were not bedridden and four had advanced dementia. Five needed a bathroom chair or bunk for showering. However, none of the differences between carriers and non-carriers or between Ward A and other wards reached statistical significance (Table).
To evaluate risk factors for KPC-KP carriage, we included the following variables in the multivariable model: age, McCabe 3, any antimicrobial at the screening day, admission from tertiary care, immunosuppression, chronic wound, length of stay in the primary care hospital, incontinence in stools, urinary catheter, wheel chair, private toileting, room size 3 or more, and any antimicrobials within three months before the screening day, but none of them was an independent explanatory variable for KPC-KP-carriage.

\section{Discussion}

The KPC-KP outbreak described here involved nine patients with asymptomatic colonisation and has been the largest in Finland so far. The outbreak was confined to one geriatric ward. The fact that the index case was found by chance in a clinical culture taken without prescription and that no direct link to travel abroad was detected, suggests the possibility that hidden colonisations may also be present elsewhere. That the outbreak ward was a dedicated cohort ward for patients with carriage of MDROs was only a coincidence, but it may have improved the containment of the outbreak because the staff was familiar with contact precautions. Rapid and extensive screening together with cohorting and isolating carriers and exposed patients were other essential tools in controlling the outbreak.

KPC-KP strain ST 512 was probably imported to Finland by an unrecognised infected or colonised patient. All CPE strains (Escherichia coli and K. pneumoniae) detected in Finland are being typed using MLST, but ST512 had not been detected in Finland before. After the 2013 outbreak, two single ST512 isolates were found in Northern Finland, in August 2013 and January 2014, also without obvious link to abroad, one in a tertiary care and the other in a primary care hospital (data not shown). This is another indication of hidden transmission of ST512 in Finland or of importation by several patients. ST512 is a single-locus variant of the widely spread KPC-2 ST258 clone. It was first described in Israel among isolates producing KPC-3 enzyme [16]. Later it caused outbreaks in the Czech Republic among hospital patients repatriated from Italy [17].

Routine screening for MDROs in Finland applies to patients repatriated from hospitals in other countries and asylum seekers needing hospital care. Screening also covers all patients seeking hospital care with a history of previous hospitalisation in another country in the preceding year. The screening covers MRSA, vancomycin-resistant enterococci (VRE) and multidrug-resistant gram-negative rods, including extended-spectrum beta-lactamase producers (ESBL) and CPE. In addition, patients exposed in Finnish hospitals (roommates of newly identified carriers or patients staying on the affected ward during an outbreak) are also screened. In an outbreak situation, the exposed patients are screened immediately, during their hospital stay. If they have already been discharged home, infection control 
nurses either contact and screen the patients as soon as the exposure becomes evident or flag the patients in the electronic patient charts to be screened at the next admission, depending on the available resources and the characteristics of the outbreak. More universal screening for multidrug-resistant gram-negative rods is not routinely performed in Finland.

In our epidemiological situation with no previous domestic CPE outbreaks, we decided to screen the whole ward after detecting a single nosocomial patient with KPC-KP. After it became clear that it was an outbreak, we chose to start multiple follow-up screenings, as recommended by Finnish guidelines, the European Centre for Disease Prevention and Control and the US Centers for Disease Control and Prevention in this type of setting $[3,18,19]$.

KPC-KP carriers were placed in contact isolation and remained there even after negative results in follow-up cultures. Also patients who were considered exposed were pre-emptively isolated until carriage was ruled out. At the outbreak ward, the annual alcohol hand rub use increased by $50 \%$, to more than $40 \mathrm{~L}$ per 1,000 patient-days, which is generally considered good, allowing at least six safe visits per patient per day (3 $\mathrm{mL}$ before and after a touch). Screening, contact precautions, cohorting and cleaning have been the key factors for successful control in several outbreak settings $[6,7,20]$. Some hospitals have also used chlorhexidine bathing for patients as well as screening of staff, although no cases were detected on that occasion $[7,20]$. In endemic settings, for instance in Israel, national interventions including active surveillance, carrier isolation and cohorting of patients and staff have shown to be effective [21].

Despite the control measures, we detected new KPC-KP carriers in consecutive screenings for almost one month after the detection of the index case. In our experience, only one set of specimens, including faeces or rectal swab, wound swab and catheter urine, was not enough to rule out KPC-KP carriage. This may be due to lack of compliance with the control measures and ongoing transmission and/or to low sensitivity of the KPC-KP screening. In addition, most patients did not have any wounds or urinary catheters and therefore submitted only faecal or rectal swabs. However, rectal cultures have been suggested to be the most sensitive single method for detection of CPE colonisation [22]. The follow-up culturing further suggested that carriage can be intermittent or short-lived, which has also been shown elsewhere [21].

Antibiotic selection pressure has been shown to have an impact on the spread of KPC-KP [7]. In our study, one third of all patients in the hospital were on antimicrobial treatment at the time of the chart review and two thirds had received antimicrobials within the preceding three months. Risk factor analysis also revealed a tendency that recent antimicrobial use may be a risk
TABLE

Characteristics of patients screened for Klebsiella pneumoniae carbapenemase-producing K. pneumoniae, Finland, $2013(\mathrm{n}=142)$

\begin{tabular}{|c|c|c|}
\hline & $\mathrm{KPC}-\mathrm{KP}(\mathrm{n}=9)$ & $\begin{array}{l}\text { No KPC-KP } \\
(n=133)\end{array}$ \\
\hline & Time (SD) & Time (SD) \\
\hline Mean age (years) & $83(S D$ 11.4) & 76 (SD 11.1) \\
\hline LOS primary care hospital (days) & $81(S D 54.2)$ & $53(S D 74.9)$ \\
\hline LOS total hospitalisation (days) & 101 (SD 57.8) & 88 (SD 96.1) \\
\hline \multirow[t]{2}{*}{$\begin{array}{l}\text { Days since last antimicrobial } \\
\text { treatment (mean) }\end{array}$} & $40(S D$ 17.4) & 77 (SD 167.0) \\
\hline & $\mathrm{n}$ & $\mathrm{n}$ \\
\hline Male sex & 3 & 60 \\
\hline Previous ESBL carriage & 0 & 9 \\
\hline Admission from tertiary care & 6 & 98 \\
\hline $\begin{array}{l}\text { Visit to tertiary care hospital } \\
\text { during stay }\end{array}$ & 3 & 29 \\
\hline $\begin{array}{l}\text { Surgery at tertiary care during } \\
\text { hospitalisation }\end{array}$ & 1 & 42 \\
\hline Room with $\geq 3$ beds & 8 & 82 \\
\hline Ward change during stay & 0 & 5 \\
\hline Room change during stay & 4 & 62 \\
\hline $\begin{array}{l}\text { Any antimicrobial treatment on } \\
\text { study day }\end{array}$ & 3 & 45 \\
\hline $\begin{array}{l}\text { Cephalosporin treatment on } \\
\text { study day }\end{array}$ & 2 & 16 \\
\hline $\begin{array}{l}\text { Fluoroquinolone treatment on } \\
\text { study day }\end{array}$ & 0 & 13 \\
\hline $\begin{array}{l}\text { Carbapenem treatment on study } \\
\text { day }\end{array}$ & 0 & 0 \\
\hline $\begin{array}{l}\text { Other antimicrobial treatment } \\
\text { on study day }\end{array}$ & 2 & 21 \\
\hline $\begin{array}{l}\text { Antimicrobial treatment within } \\
\text { last three months }\end{array}$ & 9 & 90 \\
\hline $\begin{array}{l}\text { Carbapenem treatment within } \\
\text { last three months }\end{array}$ & 1 & 8 \\
\hline McCabe1 & 1 & 12 \\
\hline McCabe2 & 6 & 99 \\
\hline McCabe3 & 2 & 22 \\
\hline Immunosuppression & 1 & 12 \\
\hline Dementia & 4 & 62 \\
\hline Bedridden/wheelchair & 3 & 74 \\
\hline $\begin{array}{l}\text { Did not need assistance in the } \\
\text { toilet }\end{array}$ & 4 & 44 \\
\hline Stool incontinence & 4 & 56 \\
\hline Chronic wound & 2 & 31 \\
\hline Urinary catheter & 3 & 24 \\
\hline
\end{tabular}

ESBL: extended-spectrum beta-lactamase producers; KPC-KP: Klebsiella pneumoniae carbapenemase-producing $K$. pneumoniae; LOS: length of stay; SD: standard deviation.

None of the characteristics covered in this Table were significantly associated with carriage of KPC-KP.

a One patient received two antibiotics. 
for KPC-KP carriage. This underlines the importance of prudent antimicrobial use in long-term care. One fifth of the treatments were considered inappropriate by three reviewing doctors in that they were prescribed for colonisation or for unspecific symptoms or for too long.

In Europe, most KPC-KP outbreak reports are from acute care hospitals, e.g. in France, Germany and Greece, where up to $30-70 \%$ of case-patients had clinical infections $[6,8,9,23]$. In our outbreak, the lack of infections may also have been due to early detection of the outbreak and to the level of care: no invasive procedures, mechanical ventilation or dialysis were provided at the primary care hospital. The index case was asymptomatic when the urine culture was taken by the nurse without a physician's prescription. Culturing asymptomatic bacteriuria or uninfected wounds is usually discouraged because it may lead to inappropriate antibiotic use. This recommendation may now need to be seen in a different light if we suspect hidden MDRO-colonisations in Finland: we may even need to encourage staff on long-term care wards to culture uninfected wounds and catheter urine regularly, just to screen for resistance, because true clinical infections caused by these pathogens are not common in long-term care. This, together with continuous training on antibiotic policy, may serve early detection of MDRO. It also conforms with the policy of this particular hospital. Earlier, the hospital had made exceptional efforts in routine universal MRSA screening, with only few cases detected, and now wants to focus on other MDROs. In addition, long-term care facilities can become reservoirs of MDRO due to the presence of several risk factors for spread such as dementia and stool incontinence.

\section{Conflict of interest}

None declared.

\section{Authors' contributions}

Mari Kanerva: outbreak investigations, manuscript writing. Kirsi Skogberg: outbreak investigations, manuscript writing. Kaisa Ryynänen: outbreak investigations. Anita Pahkamäki: outbreak investigations. Jari Jalava: MLST and PGFE typing, manuscript writing. Jukka Ollgren: statistics. Eveliina Tarkka: detection of bacterial isolates and manuscrip writing. Outi Lyytikäinen: study design, manuscript writing.

\section{References}

1. Nordmann P, Naas T, Poirel L. Global spread of carbapenemase-producing Enterobacteriaceae. Emerg Infect Dis. 2011;17(10):1791-8. http://dx.doi.org/10.3201/ eid1710.110655 PMID:22000347

2. Munoz-Price LS, Poirel L, Bonomo RA, Schwaber MJ, Daikos GL, Cormican M, et al. Clinical epidemiology of the global expansion of Klebsiella pneumoniae carbapenemases. Lancet Infect Dis. 2013;13(9):785-96. http://dx.doi.org/10.1016/S14733099(13)70190-7 PMID:23969216</

3. European Centre for Disease Prevention and Control (ECDC). Risk assessment on the spread of carbapenemase-producing Enterobacteriaceae (CPE) through patient transfer between healthcare facilities, with special emphasis on cross-border transfer. Stockholm: ECDC; 2011. ISBN 978-92-9193-317-4.
Available from: http://ecdc.europa.eu/en/publications/ Publications/110913 Risk_assessment_resistant_CPE.pdf. doi:10.2900/59034

4. Lin MY, Lyles-Banks RD, Lolans K, Hines DW, Spear JB, Petrak $\mathrm{R}$, et al. The importance of long-term acute care hospitals in the regional epidemiology of Klebsiella pneumoniae carbapenemase-producing Enterobacteriaceae. Clin Infect Dis. 2013;57(9):1246-52. http://dx.doi.org/10.1093/cid/cit50o PMID:23946222

5. Glasner C, Albiger B, Buist G, Tambić Andrasević A, Canton R, Carmeli Y, et al. Carbapenemase-producing Enterobacteriaceae in Europe: a survey among national experts from 39 countries, February 2013. Euro Surveill. 2013;18(28):20525. http://dx.doi. org/10.2807/1560-7917.ES2013.18.28.20525 PMID:23870096

6. Carbonne A, Thiolet IM, Fournier S, Fortineau N, KassisChikhani N, Boytchev I, et al. Control of a multi-hospital outbreak of KPC-producing Klebsiella pneumoniae type 2 in France, September to October 2009. Euro Surveill. 2010;15(48):19734. PMID:21144448

7. Robustillo Rodela A, Díaz-Agero Pérez C, Sanchez Sagrado T, Ruiz-Garbajosa P, Pita López MJ, Monge V. Emergence and outbreak of carbapenemase-producing KPC-3 Klebsiella pneumoniae in Spain, September 2009 to February 2010: control measures. Euro Surveill. 2012;17(7):20086. PMID:22370016

8. Steinmann J, Kaase M, Gatermann S, Popp W, Steinmann E, Damman M, et al. Outbreak due to a Klebsiella pneumoniae strain harbouring KPC-2 and VIM-1 in a German university hospital, July 2010 to January 2011. Euro Surveill. 2011;16(33):19944. PMID:21871227

9. Souli M, Galani I, Antoniadou A, Papadomichelakis E, Poulakou G. Panagea T, et al. An outbreak of infection due to betaLactamase Klebsiella pneumoniae Carbapenemase 2-producing K. pneumoniae in a Greek University Hospital: molecular characterization, epidemiology, and outcomes. Clin Infect Dis. 2010;50(3):364-73. http://dx.doi.org/10.1086/649865 PMID:20041768

10. Samuelsen $\varnothing$, Naseer U, Tofteland S, Skutlaberg DH, Onken A, Hjetland R, et al. Emergence of clonally related Klebsiella pneumoniae isolates of sequence type 258 producing plasmidmediated KPC carbapenemase in Norway and Sweden. I Antimicrob Chemother. 2009;63(4):654-8. http://dx.doi. org/10.1093/jac/dkp018 PMID:19218573

11. Österblad M, Kirveskari J, Hakanen AJ, Tissari P, Vaara M, Jalava J. Carbapenemase-producing Enterobacteriaceae in Finland: the first years (2008-11). I Antimicrob Chemother. 2012;67(12):2860-4. http://dx.doi.org/10.1093/jac/dks299 PMID:22855858

12. Tofteland S, Naseer U, Lislevand JH, Sundsfjord A, Samuelsen 0 . A long-term low-frequency hospital outbreak of KPCproducing Klebsiella pneumoniae involving Intergenus plasmid diffusion and a persisting environmental reservoir. PLoS ONE. 2013;8(3):e59015. http://dx.doi.org/10.1371/journal. pone.0059015 PMID:23536849

13. European Committee on Antimicrobial Susceptibility Testing (EUCAST). Breakpoint tables for interpretation of MICS and zone diameters, v. 3.1. Växjö: EUCAST; 2013. Available from: http://www.eucast.org/fileadmin/src/media/PDFs/ EUCAST_files/Breakpoint_tables/Breakpoint_table_v_3.1.pdf

14. Pasanen T, Koskela S, Mero S, Tarkka E, Tissari P, Vaara $M$, et al. Rapid molecular characterization of Acinetobacter baumannii clones with rep-PCR and evaluation of carbapenemase genes by new multiplex PCR in Hospital District of Helsinki and Uusimaa. PlosOne 2014;9(1):e85854 doi: 10.1371/journal.pone.0085854. eCollection 2014.

15. McCabe WR, Jackson GG. Gram-negative bacteremia. I. Etiology and ecology. Arch Intern Med. 1962;110(6):847-55. http:// dx.doi.org/10.1001/archinte.1962.03620240029006

16. Warburg G, Hidalgo-Grass C, Partridge SR, Tolmasky ME, Temper V, Moses AE, et al. A carbapenem-resistant Klebsiella pneumoniae epidemic clone in Jerusalem: sequence type 512 carrying a plasmid encoding aac(6D)-lb. J Antimicrob Chemother. 2012;67(4):898-901. http://dx.doi.org/10.1093/ $\mathrm{jac} / \mathrm{dkr} 552$ PMID:22287232

17. Hrabák J, Papagiannitsis CC, Študentová V, Jakubu V, Fridrichová M, Zemlickova H, et al. Carbapenemase-producing Klebsiella pneumoniae in the Czech Republic in 2011. Euro Surveill. 2013;18(45):20626. http://dx.doi.org/10.2807/15607917.ES2013.18.45.20626 PMID:24229789

18. Kolho E, Lyytikäinen O. Ohje moniresistenttien mikrobien tartunnantorjunnasta. [Guidelines for the control of multidrugresistant microbes]. Finnish. Helsinki: National Institute for Health and Welfare; 2014. Available from: http://www.julkari. $\mathrm{fi} /$ bitstream/handle/10024/116266/URN ISBN 978-952-302260-7.pdf?sequence $=1$

19. Centers for Disease Control and Prevention (CDC). Guidance for control of carbapenem-resistant Enterobacteriacae (CRE). 
Atlanta: CDC; 2012. Available from: http://www.cdc.gov/hai/ pdfs/cre/cre-guidance-508.pdf

20. Munoz-Price LS, Hayden MK, Lolans K, Won S, Calvert K,

Lin M, et al. Successful control of an outbreak of Klebsiella

pneumoniae carbapenemase-producing K. pneumoniae

at a long-term acute care hospital. Infect Control Hosp

Epidemiol. 2010;31(4):341-7. http://dx.doi.org/10.1086/651097 PMID:20175685

21. Schwaber MJ, Carmeli Y. An ongoing national intervention to contain the spread of carbapenem-resistant

enterobacteriaceae. Clin Infect Dis. 2014;58(5):697-703. http://

dx.doi.org/10.1093/cid/cit795 PMID:24304707

22. Thurlow CJ, Prabaker K, Lin MY, Lolans K, Weinstein RA, Hayden MK, et al. Anatomic sites of patient colonization and environmental contamination with Klebsiella pneumoniae carbapenemase-producing Enterobacteriaceae at longterm acute care hospitals. Infect Control Hosp Epidemiol. 2013;34(1):56-61. http://dx.doi.org/10.1086/668783 PMID:23221193

23. Zagorianou A, Sianou E, losifidis E, Dimou V, Protonotariou E, Miyakis S, et al. Microbiological and molecular characteristic of carbapenemase-producing Klebsiella pneumoniae endemic in a tertiary Greek hospital during 2004-2010. Euro Surveill. 2012;17(7):20088. PMID:22370015 